\title{
Histerectomía post-parto
}

\section{Clínica David Restrepo}

\author{
Dres.: Hernando Sánchez Varela, Carlos Hoyos Cruz
}

\section{INTRODUCCION}

\section{Reseña histórica}

Evolución de la cesárea histerectomía a través del tiempo.

Eduardo Porro fue el primer cirujano que efectuó cesárea con histerectomía en paciente viva hacia el año de 1876.

Joseph Cavallini de Florencia en 1768 escribió un trabajo experimental sobre operación cesárea histerectomía en animales de laboratorio.

G. P. Michaelis 1809 y James Blundell en 1823 argumentaron que podría efectuarse histerectomía cesárea en la mujer pero sus experiencias se limitaron a animales de laboratorio.

Horatio Storer 1869 ejecutó primera cesárea con histerectomía en paciente viva en los, EE.UU.

La indicación de cesárea fue tumor previo y la de la histerectomía subtotal por atonía uterina; la paciente falleció tres días después.

Eduardo Porro en 1876 describió un trabajo de tallado sobre la primera cesárea histerectomía en la que madre e hijo so- brevivieron. Inzana y Previtali en Italia y Hegar en Alemania efectuaron esta operación pocos meses después en varios casos. Todas las madres fallecieron.

En 1878, en Berna, se describió la primera modificación de la técnica de Porro.

La mortalidad materna en esta revisión fue de $58 \%$ y la supervivencia fetal $86 \%$.

Richardson en 1881, efectuó la primera cesárea histerectomía satisfactoria en los EE.UU.

Spencer Wells en 1881, de Gran Bretaña, describió la primera cesárea histerectomía total por $\mathrm{Ca}$. invasivo de cervix.

Gordon en 1884 ejecutó en Gran Bretaña la primera cesárea histerectomía satisfactoria y presentó primer informe sobre incisión uterina transversal baja.

De 1800 a 1900 la cesárea con histerectomía fue aconsejada para salvar la vida materna, pero Red en 1900 de E.U., bosquejó las siguientes indicaciones para la intervención:

1. Feto muerto e intección uterina.

2. Atresia extensa de vagina que impide la expulsión de loquios. 
3. Ci. de cuello uterino.

4. Atonía uterina o hemorragia incontrolable a nivel de placenta.

5. Ruptura uterina.

Duncan y Targett en 1900 en Gran Bretaña sugirieron histerectomía subtotal para esterilización después de operación cesárea, a pesar de la alarmante mortalidad materna.

J. W. Harris 1922 Hospital John Hopkins informó de serie de 64 casos; de los cuales 18 para esterilización, la mortalidad materna en ésta serie fue de $4.68 \%$.

Lash y Cummings 1935 propusieron las siguientes indicaciones:

1. Infección uterina

2 Pérdida aguda de sangre

3. Nefropatías crónicas

4. T.B.C.

Davis en 1951 en Chicago anota que se justifica cesárea histerectomía en pacientes cercanos al fin del período reproductivo.

Sandburg, Hallot y Hirsch en 1958 se refirieron a la conveniencia de cesárea histerectomía como método de esterilización "adelanto lógico de la cirugía obstétrica actual".

Pletsch y Sandberg en 1963 resumieron cl debate sobre cesárea histerectomía y sus indicaciones con estadísticas.

Brennere en 1964 concluye que no se recomienda la cesárea histerectomía con fines de esterilización por los riesgos que ella representa.

Barclay y Mickal consideran que en ciertas situaciones pueden justificarse los métodos electivos, que es factible llevar a cabo con escaso margen de riesgo.

\section{MATERIALES Y METODOS}

Los datos del presente estudio se obtuvieron del archivo obstétrico de la Clínica de Maternidad David Restrepo.

Se revisaron las historias clínicas de 32 años comprendidos entre el mes de julio de 1952 y el mes de julio de 1985.

En estc lapso se atendieron un total de 88.621 partos; dentro de los cuales espontáneos fueron 61.733; intervenidos (fórceps, espátulas) 11.455 que corresponden al $12.92 \%$; y cesáreas 15.433 que representa el $17.41 \%$ del total.

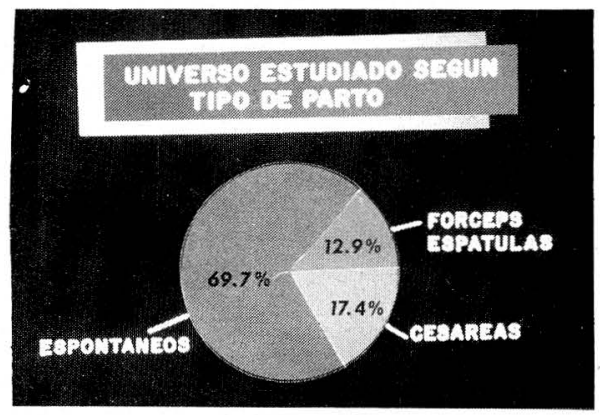

Del número total de partos 88.621 , se presentaron 44 casos de histerectomía post parto, de los cuales post parto vaginal 5 casos que significan 1 por cada 12.347 partos espontáneos atendidos; post parto intervenido (fórceps) 3 casos, que representan 1 por cada 3.818 partos atendidos con fúrceps; $y$ posteriores a cesárea 36 casos, lo que representa 1 de cada 429 cesáreas efectuadas. Sólo se tuvieron en cuenta para ser incluídos dentro del estudio aquellas pacientes cuya edad gestacional fuera superior a 27 scmanas.

Clasificamos las histerectomías post parto en indicadas y clectivas. Para clasificarlas como indicadas tuvimos en cuenta 2 parámetros fundamentales:

1. Las histerectomías de urgencia que se llevaron a cabo para cohibir hemorra- 
gia que pusiera en peligro la vida de la madre, como por ejemplo: atonía uterina, ruptura uterina $y$ acretismo placentario, etc.

2. Las histerectomías efectuadas por infección, básicamente endometritis, miometritis, endomiometritis, peritonitis, sepsis.

Para clasificarlas como electivas se consideraron las no comprendidas dentro del grupo anterior (indicadas) como por ejemplo: cicatriz uterina defectuosa, tumor previo, miomatosis uterina, cáncer, prolapso genital, etc.

Encontramos dentro de los 44 casos de histerectomía post parto, 37 casos de histerectomía indicada, que correspondieron al $84.09 \%$ y 7 casos de histerectomías electivas que significan el $15.91 \%$.

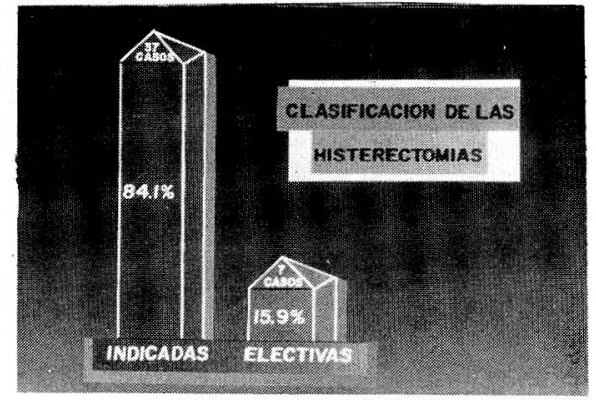

\section{Edad y Paridad}

En el cuadro correspondiente se resume la distribución por edades de las pacientes en quienes fue necesario practicar histerectomía post parto.

De los 44 casos hallados, se encontraban dentro de los 21 y 30 años, 16 que representan el $36.36 \%$; entre los 31 y 40 años 24 casos el $54.53 \%$ y de 41 a 50 años 4 casos que son el $9.08 \%$.

La menor edad hallada fue de 21 años en dos pacientes, una de ellas G 111 con una cesárea anterior.

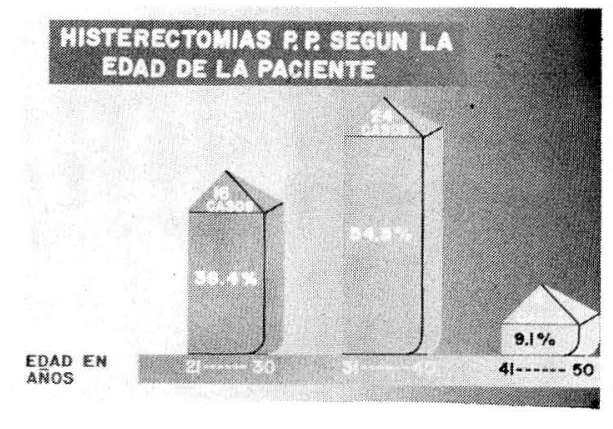

Dentro de éste grupo de edades (21 a 30 años), 16 casos, sólo cinco tenían antecedente de cesárea, tres casos una cesárea anterior y dos tenían dos cesáreas anteriores. Es importante resaltar que la indicación de histerectomía tuvo relación con la cesárea en cuatro de los cinco casos (segmento uterino defectuoso y acretismo placentario).

El mayor número de casos (24) se encontraron en el grupo de edad de 31 a 40 años; de éstos se encontró el antecedente de cesárea en 12 pacientes, de los cuales la indicación de histerectomía se relacionó con el antecedente de cesárea en 9 casos (ruptura uterina y acretismo placentario).

La mayor edad hallada en la revisión fue de una señora de 47 años G XIII quien terminó en histerectomía por ruptura uterina.

En este grupo de edad de 41 a 50 años sólo se presentaron 4 casos, uno de ellos con antecedente de tres cesáreas anteriores e histerectomía por ruptura uterina

Entre primigestantes se encontraron de las 44 pacientes, ocho, que oscilaron entre los 21 y 42 años, todas ellas fueron por cesárea con indicación diversa y la histerectomía se motivó, por atonía uterina 4 casos, por miometritis 2 casos y una por $\mathrm{Ca}$. de ovario $\mathrm{y}$ otro por miomatosis uterina. 


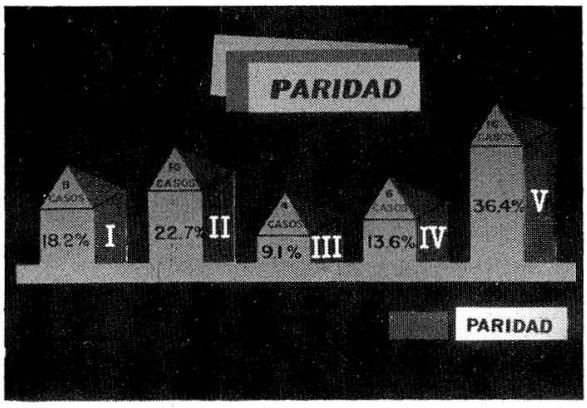

En el grupo de pacientes de paridad de 5 ó más, se encontraron 16 casos, la mayor paridad en 2 señoras G XIII como indicación de histerectomía 8 por ruptura uterina, de las cuales dos tenían antecedente de cesárea; los restantes se atribuyen a multiparidad y/o uso de fórceps.

\section{Partos espontáneos o Fórceps e histerectomía}

Se encontraron de los 44 casos de histerectomía post parto: 5 espontáneos y tres intervenidos con fórceps.

De los espontáneos 3 casos en presentación de pelvis y 2 en presentación cefálica. Dos de presentación de pelvis fueron por ruptura uterina en grandes multíparas y el otro por atonía uterina.

En presentación cefálica dos: uno de ellos por ruptura uterina debida a maniobra de Kristeller y el otro por miometritis.

De los intervenidos los tres por aplicación de fórceps; un fórceps medio en cefálica y dos fórceps por retención de cabeza última en presentación de pelvis, la histerectomía fue indicada en los tres por ruptura uterina.

\section{Indicación de cesárea}

De las 44 histerectomías post parto, 36 se efectuaron post operación cesárea que corresponde al $81.81 \%$.
Es de tener en cuenta que figura como indicación de cesárea aquella que verdaderamente decidió la intervención; como por ejemplo en el caso de cesárea anterior y sufrimiento fetal agudo; la indicación de cesárea fue el S.F.A. No así en los casos en los cuales se presentaban dos o más cesáreas anteriores en cuyo caso la indicación fue la Iterativez.

Con la anterior aclaración la mayor causa indicación de cesárea que terminó en histerectomía (indicada o electiva) fue la Iterativa en 10 casos, seguida de placenta previa en 5 casos y D.C.P. en 3 casos. Otras indicaciones fueron situación transversa, presentación de pelvis, S.F.A., ruptura uterina, distocia dinámica de contracción y distocia de cuello cada una de ellas con dos casos.

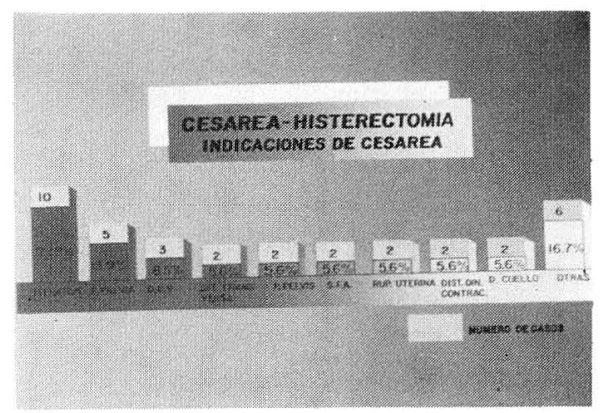

Las demás indicaciones se resumen en el cuadro correspondiente.

\section{Clasificación de Histerectomías Post parto}

Algunos de los autores consultados sobre el tema consideran como indicadas las histerectomías de urgencia por hemorragia incontrolable y como electivas las demás. Nosotros consideramos conveniente incluir dentro de las indicadas además de las de urgencia por hemorragia las realizadas por infección, por considerarlas igualmente de urgencia. Indicadas fueron $37(84.09 \%$ !) y electivas $7(15.91 \%)$. 
Las histerectomías indicadas por hemorragia fueron el mayor número, por infección solamente 5 casos.

Dentro de las indicadas por hemorragia sobresale el acretismo placentario 15 casos; seguidos de ruptura uterina y atonía uterina con 10 casos cada uno.

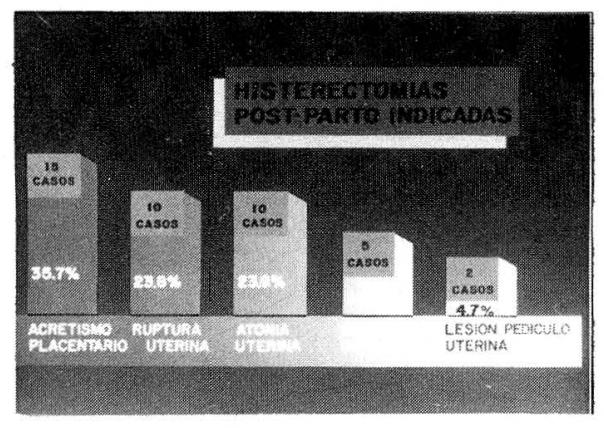

El acretismo placentario coincidió con cicatriz uterina (cesárea o cesáreas anteriores) en 11 de los 15 casos.

La asociación de placenta acreta, placenta previa y antecedente de cesárea se vio en 7 casos, con un especial incremento en los últimos cinco años.

Se presentaron combinaciones dentro de las indicadas por hemorragia en cinco casos de las historias revisadas.

Atonía uterina más placenta acreta: 3

Ruptura uterina más placenta acreta: 2

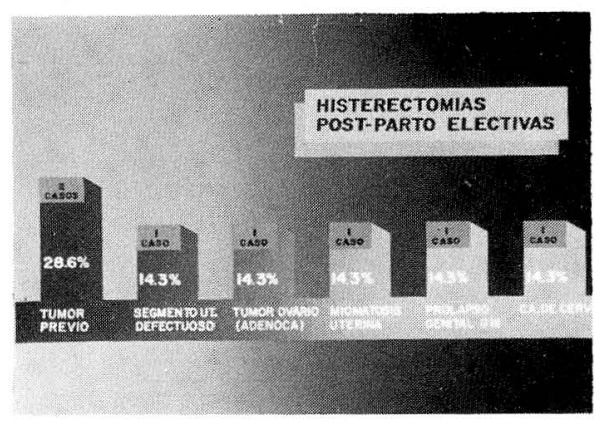

Por esta razón el número de indicadas supera el real; pues cada una de éstas combinaciones fue tenida en cuenta por separado.

Las electivas 7 casos (15.91\%), se registran en el cuadro adjunto.

\section{Tipo de intervención}

Hacemos referencia al tipo de cirugía realizada, es decir, si la histerectomía fue total o subtotal; deseando "conocer la tendencia del especialista en la Clínica David Restrepo al respecto.

No consideramos necesario describir la técnica quirúrgica de la histerectomía por cuanto se ciñe a la técnica habitual internacionalmente conocida.

De las 44 histerectomías post parto halladas en nuestra revisión encontramos la siguiente distribución:

Histerectom ías subtotales 13 (29.54\%) Histerectomías totales $31(70.45 \%)$

De las histerectomías subtotales 12 correspondieron a histerectomías indicadas o de urgencia y una histerectomía electiva por tumor previo (mioma); la razón primordial en estos casos fue la de acortar al máximo el tiempo quirúrgico (tiempo anestésico) por mal estado de la paciente y en otros, pocos casos, por dificultad técnica relacionados éstos con infección (peritonitis) en tres casos.

Debemos anotar que cuatro de estas histerectomías subtotales se hicieron por placenta acreta y la indicación de cesárea fue placenta previa; hecho al que hacemos referencia posteriormente.*

\section{Histerectomía mediata o inmediata}

Consideramos como inmediata aqueIlas histerectomías que se realizaron inme- 


\section{Tipo de intervención*}

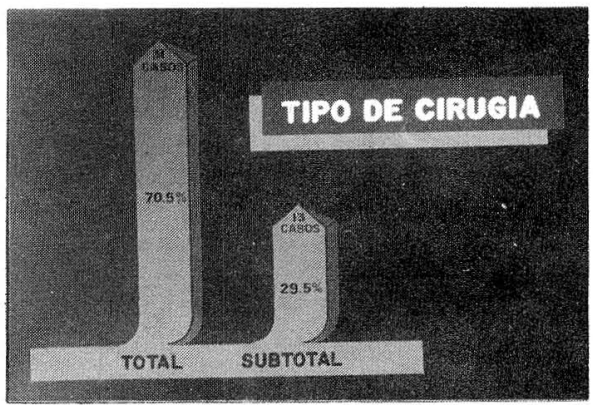

diatamente posterior al parto o durante el mismo acto operatorio de la cesárea, o en las primeras 12 horas; y como mediata las practicadas después de éste tiempo.

Inmediatas fueron $37(84.09 \%)$ y mediatas 7 (15.91\%).

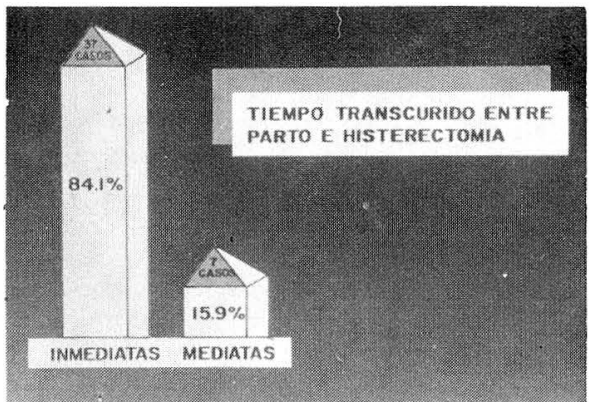

De las inmediatas 26 se efectuaron dentro del mismo acto operatorio de la cesárea o inmediatamente post parto.

De las mediatas la de mayor tiempo transcurrido entre parto e histerectomía fue de 31 días por hemorragia atribuida a retención de restos y la de menor tiempo de 31 horas por atonía uterina en pacientes con E.V.H.C. y pre-eclampsia.

Todos los casos de histerectomía indicada por infección fueron mediatas variando de 4 a 30 días de intervalo.

Todas las mediatas fueron histerectomías indicadas.

\section{Intervención concomitante}

La mayoría de las intervenciones concomitantes fueron ginecológicas; S.O.U., S.O.B., salpinguectomía unilateral, bilateral y ooforectomía. Solamente dos casos no lo fueron, siendo la intervención herniorrafia umbilical y omentectomía por Ca. de ovario.

\section{Complicación Post operatoria}

Se presentaron 3 casos de infección de la herida quirúrgica; dos de ellos leves y el otro con necesidad de drenaje por formación de absceso y dehiscencia; como es lógico suponer guardaron relación directa con el estado patológico primario (amnionitis, peritonitis).

En tres pacientes se diagnosticó hemorragia intra abdominal, dos fueron reintervenidos encontrándose en uno pedículo utero-ovárico sangrante el cual fue ligado y el otro correspondió a una histerectomía subtotal con sangrado de arteria cervical por lo cual se completó histerectomía con extracción de cuello.

En la otra paciente el diagnóstico fue tardío y falıeció por bematoma infectado y sepsis.

Los dos casos de fístula vesico vaginal se debieron a lesión inadvertida de vejiga durante la intervención, siendo corregidas con éxito posteriormente.

El absceso de cúpula correspondió a un caso de panmetritis en el que se practicó el drenaje de 2.000 cc de material purulento y fue necesario completar histerectomía con extracción de cuello.

\section{Uso de antibióticos}

Se suministraron antibióticos en todos los casos, en algunos profilácticos y en otros curativos; estos últimos fueron em- 
pleados en aquellos casos en los cuales la histerectomía fue indicada por infección. El tipo de antibiótico utilizado dependió de la época de su empleo. La asociación antibiótica más frecuentemente suministrada fue ampicilina-gentamicina.

\section{Transfusión}

Fue necesaria transfusión en 39 de las 44 pacientes sometidas a histerectomía post parto. Variando de $500 \mathrm{cc}$ a 13.500 Cc de sangre fresca total; éste último en uno de los casos de coagulopatía de consumo en una mujer de 34 años.

\section{Permanencia hospitalaria post histerectomía}

El tiempo de permanencia post operatoria (post histerectomía) promedio fue de 11.3 días. Estas cifras incluyen todos los casos y no se corrigieron para excluir pacientes excepcionales que exigieron hospitalización duradera.

En el grupo de histerectomía indicada por hemorragia o infección el promedio de hospitalización fue de 12 días; en el electivo de 9.2 días. La hospitalización después de la histerectomía post parto no complicada suele ser superior que tras la operación cesárea sin complicación.

\section{Morbilidad Post histerectomía}

En 11 pacientes equivalentes al $25 \%$ se presentó morbilidad post operatoria. Es-

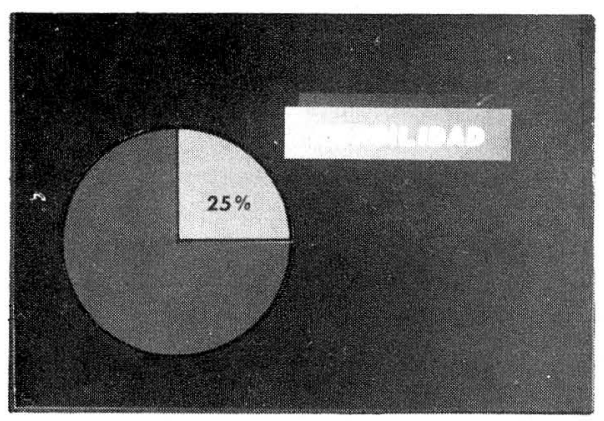

tas complicaciones que fueron shock hipovolémico, infección urinaria, coagulopatía de consumo, tromboflebitis y sepsis se esquematizan en el cuadro correspondiente.

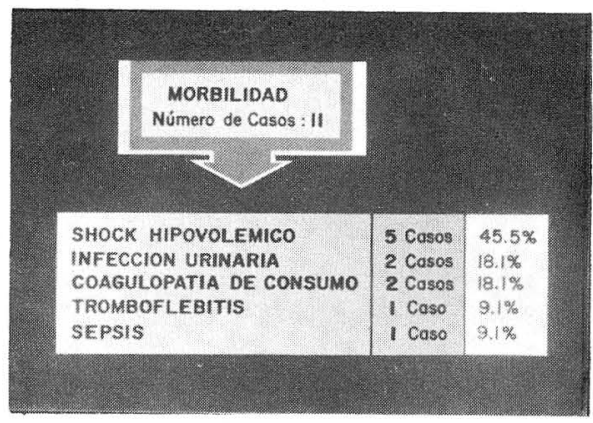

\section{Mortalidad Materna:}

Ocurrieron dos muertes en nuestra revisión de histerectomía post parto en la Clínica David Restrepo.
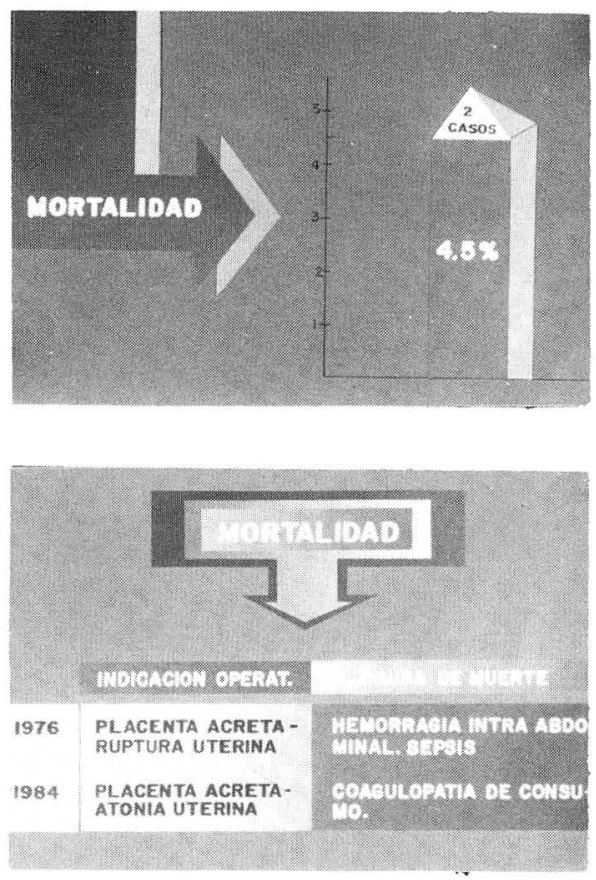
La primera en el año de 1976 y la segunda en 1984. Las indicaciones de histerectomía fueron placenta acreta y ruptura uterina en el primero y placenta acreta $y$ atonía uterina en el segundo.

La causa de muerte fue hemorragia in. tra abdominal sepsis y coagulopatía de consumo respectivamente.

\section{Esta mortalidad representa el $4.5 \%$.}

La primera paciente de 34 años gestante $\mathrm{V}$, todos los partos por cesárea, falleció 8 días después de la histerectomía.

La otra paciente de 37 años, igualmente multigestante $\mathrm{V}$, sin antecedentes de cesárea, falleció 1 hora 30 minutos post histerectomía.

\section{COMENTARIOS Y CONCLUSIONES}

La historia de la histerectomía post parto en la Clínica de Maternidad David Restrepo de Bogotá se revisó retrospectivamente al tabular las indicaciones, complicaciones y resultados de 44 operaciones (histerectomia post parto) en un período de 32 años, de un total de 88.621 partos atendidos en este lapso.

La frecuencia de histerectomía post parto en la Clínica es sumamente baja, si la comparamos con la hallada entre otros autores como Ramírez y Real que repor$\tan 29$ casos en un período de cinco años y Urzúa y Marín quienes encontraron 45 casos en dos años.

Coincidimos con estas publicaciones de trabajos realizados en el Hospital General, Sistema de Salud y Salubridad de México D.F. por los doctores Ramírez y Real y del Instituto de Seguro Social de México D.F. por los Doctores Urzúa y Marín en varios de los parámetros evaluados y sus resultados (distribución por edades, paridad, indicaciones de cesárea e histerectomía); siendo la morbilidad menor en nuestro estudio que fue del $25 \%$, comparado con el $42 \%$ en la revisión de Urzúa.

Igualmente compartimos con ellos la posición de efectuar este tipo de intervención (histerectomía) preferencialmente en situaciones indicadas o absolutas por hemorragia o infección y en contados casos como procedimiento electivo o relativo.

No ju stificamos realizar el procedimiento con fines de esterilización como lo preconizan investigadores como Barclay y Easterday en los hospitales de Nueva Orleans y Boston de los E.U.; por cuanto aunque algunos reportan ínfima morbilidad, la mayoría da cifras del 10 al 40\%.

Creemos conveniente ser prudentes en la determinación de histerectomía en los casos de hemorragia o infección, pero una vez agotados los recursos para controlar estos procesos la decisión debe ser oportuna, sin entrar en consideraciones de edad o paridad de la paciente.

Es de tener en cuenta que en la histerectomía post parto o cesárea histerectomía el aumento del riesgo sanguíneo, la anatomía deformada y la fragilidad de los tejidos, predisponen a defectos de hemostasıa y que las adherencias de cesáreas previas aumentan el número de lesiones vesicales y por ende la morbilidad.

Concluimos por los resultados que la morbilidad y permanencia hospitalaria consecutiva a operación cesárea histerectomía supera a la cesárea exclusiva.

La histerectomía en casos de placenta previa y ácreta debe ser total, por cuanto con la subtotal se corre el riesgo de sangrado post operatorio; debido a la gran circulación colateral desarrollada por las arterias cervico vaginales en el cuello uterino en estos casos; lo mismo que en infección debe ser total si es posible, a fin de extirpar completamente el foco infeccioso. 
La indicación sobresaliente de histerectomía en los primeros años de la revisión fue la ruptura utcrina, en relación con la multiparidad, el uso de fórceps y otras maniobras de extracción fetal.

Por el contrario el mayor número de histerectomías indicadas por hemorragia se presentó en los últimos cinco años, en pacientes con antecedentes de cesárea; y de la estrecha relación que encontramos entre cesárea, placenta previa y acretismo placentario, decidimos revisar las indicaciones de cesárea, decisión progresivamente creciente y a considerar y revaluar la determinación de cesárea, en caso de cesárea anterior. Està es sin duda la conclusión más importante de nuestro trabajo.

\section{RESUMEN}

Encontramos en un total de 88.621 partos atendidos de julio de 1952 a julio de 1985, 44 pacientes en quienes se practicó histerectomía post parto; de las cuales 36 posteriores a cesárea y 8 post parto espontáneo o intervenido por fórceps.

La mayor frecuencia de edad fue hallada en el grupo de 30 a 40 años.

El mayor número de casos en cuanto a paridad se encontró en multigestantes (36.36\%). Histerectomía total fue realiza-

\section{BIBLIOGRAFIA}

1. DURFEE, R.B.: Evolución de la cesárea histerectomía, Clín. Obstet. Ginecol. 12: 575, 1969.

2. BARCLAY, D.L.: Cesárea histerectomía en el Hospital Charity de Nueva Orleans: 1000 operaciones consecutivas. Clin. Obstet. Ginecol. 12: 635, 1969. da en 31 casos $(70.45 \%)$. La mayor indicación de cesárea que terminó en histerectomía fue la Iterativa.

Clasificamos las histerectomías en indicadas y electivas, las indicadas por hemorragia o infección representaron el $84 \%$, sobresaliendo el acretismo placentario, lá ruptura y atonía uterina.

Sólo se presentaron cinco casos de infección (endomiometritis).

Histerectomías inmediatas que se realizaron dentro de las primeras 12 horas post parto o post cesárea significaron el $84 \%$.

La complicación post operatoria más frecuente fue le infección de herida quirúrgica y hemorragia intra abdominal. Se presentaron en esta revisión dos fallecimientos $(4.54 \%)$ atribuyéndose la causa de muerte a hemorragia intra abdominal, sepsis y coagulopatía de consumo. Los hallazgos del presente estudio coinciden con otras publicaciones sobre el tema.

El acretismo placentario y la atonía uterina son la primera indicación de histerectomía precedidos de cesárea o cesáreas anteriores, especialmente en los últimos cinco años; lo que nos lleva a considerar y revisar las indicaciones de cesárea y a revaluar el aforismo de cesárea anterior igual cesárea.

3. BARCLAY, D.L.: Cesarean-Histerectomy, Thirty Years Experience. Obstet. Gynecol. 35:120, 1970.

4. MORTON J. H,: Cesarean Hysterectomy. Amer. J. Obstet. Gynec., 83: $1422,1962$.

5. DYER, I.: Total Cesarean and Puerperal Hysterectomy. Obstet, and Gynec., 9: 696, 1957. 
6. PLETSCH, T.D. y SANDBERG, E.C.: Cesarean Hysterectomy for sterilization. Amer. J. Obstet. Gynec. 85: $254,1963$.

7. DAVIS, M.E.: Complete Cesarean Hysterectomy; logical advance in modern obstetric surgery. Amer. J. Obstet. Gynec. 62: 838, 1951.

8. JAMES, D. R, y col.: Cesarean Hysterectomy. Min. Med., 51: 17, 1968.

9. SCHNEIDER, G.T.: Cesarean Total Histerectomy J. La. Med. Soc., 111: $456,1959$.

10. EASTERDAY, C.H.: Cesarean Hysterectomy at the Boston Hospital for Women. Clin. Obstet. Gynecol. 12: $652,1969$.

11. RUIZ VELASCO, V., ROSAS, A. J. y RIO DE LA LOZA, L. F.: Cesárea histerectomia Ginec. Obstet. Mex. 22: 591; 1967.
12. CHAVEZ AZUELA J.:Morbimortalidad materna en la cesárea histerectomía. Ginec. Obstet. Mex. 35: 511, 1974.

13. LARSSON, D.: Evaluation of Cesarean Section Hysterectomy J. Amer. Med. Wom. Ass., 17: 720, 1962.

14. DE COSTA, E. J.: Cesarean Section Clin. Obstet. Gynec., 2: 937-1048, 1960.

15. OLEARY, J. A.: Cesarean Hysterectomy: a 15 year review. J. Reprod. Méd. 4: 231, 1970.

16. ARANA, A., J.A.: Cesárea Histerectomía Total. Reporte de 100 casos consecutivos y revisión de la estadística mundial. Ginec. Obstet. Mex. 40: 419, 1976.

17. URZUA, M. A. y MARIN, R.A.: Cesárea Histerectomía. Ginec. Obstet. Méx. 48: 288, 1980.

18. RAMIREZ, C.J. y REAL A.G.: Cesárea Histerectomía. Ginec. Obstet. Méx. 48: 288, 1980.

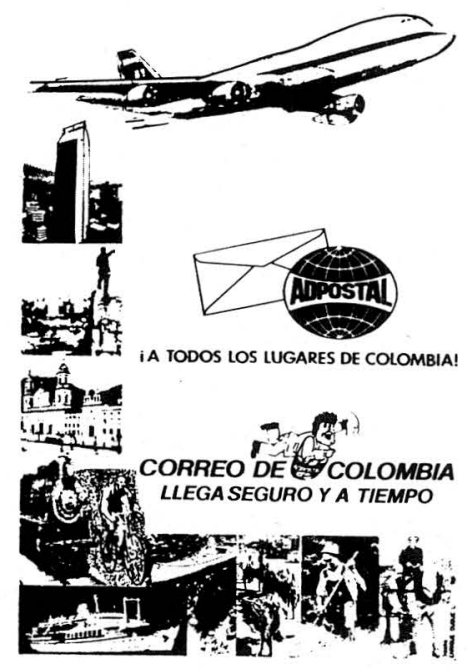

